\title{
UNIVERSITYOF
}

FORWARD

THINKING

WESTMINSTER用

WestminsterResearch

http://www.westminster.ac.uk/westminsterresearch

\section{Empirische Forschung zur Verbraucherschlichtung}

Zwischenbericht zum Forschungsvorhaben „Funktionsweise der

Allgemeinen Verbraucherschlichtungsstelle" im Auftrag des

BMJV

Creutzfeldt, N. and Steffek, F.

This is a copy of the final version of an article published in Zeitschrift für Konfliktmanagement, 22 (2), pp. 40-44.

It is available from the publisher at:

https://dx.doi.org/10.9785/zkm-2019-220203

The WestminsterResearch online digital archive at the University of Westminster aims to make the research output of the University available to a wider audience. Copyright and Moral Rights remain with the authors and/or copyright owners.

Whilst further distribution of specific materials from within this archive is forbidden, you may freely distribute the URL of WestminsterResearch: ((http://westminsterresearch.wmin.ac.uk/).

In case of abuse or copyright appearing without permission e-mail repository@westminster.ac.uk 


\section{Naomi Creutzfeldt und Felix Steffek}

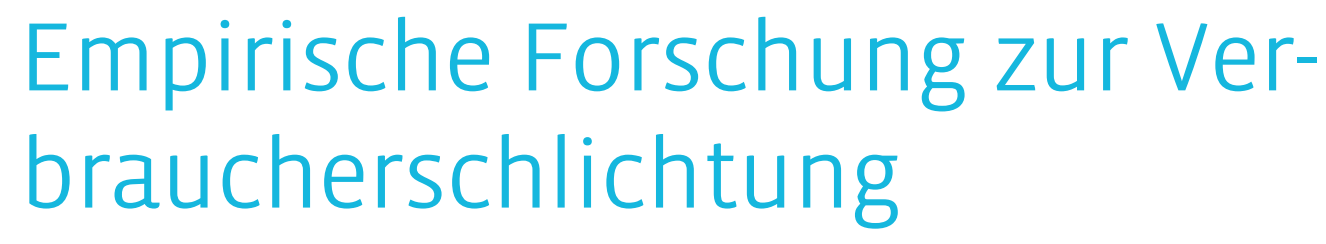

\section{Zwischenbericht zum Forschungsvorhaben „Funktionsweise der All- gemeinen Verbraucherschlichtungsstelle“ im Auftrag des BMJV}

Das Bundesministerium der Justiz und für Verbraucherschutz (BMJV) hat zur Vorbereitung von Reformen auf dem Gebiet der Verbraucherstreitbeilegung ein empirisches Forschungsvorhaben in Auftrag gegeben. Die Studie „Funktionsweise der Allgemeinen Verbraucherschlichtungsstelle“ untersucht die Schlichtungswirklichkeit der Allgemeinen Verbraucherschlichtungsstelle am Zentrum für Schlichtung e.V. in Kehl. Im Dezember 2018 hat die Bundesregierung einen Zwischenbericht über das Forschungsvorhaben sowohl dem Bundestag als auch dem Bundesrat vorgelegt. Der vorliegende Beitrag fasst die wichtigsten Ergebnisse des Berichts zusammen.

\section{I. Überblick}

Das Bundesministerium der Justiz und für Verbraucherschutz (BMJV) fördert gem. $\$ 43$ Abs. 1 Verbraucherstreitbeilegungsgesetz (VSBG) bis zum 31.12.2019 die Arbeit einer ausgewählten Allgemeinen Verbraucherschlichtungsstelle, die bundesweit tätig ist. Dabei handelt es sich um die Allgemeine Verbraucherschlichtungsstelle am Zentrum für Schlichtung e.V. in Kehl, die ihre Tätigkeit am 1.4.2016, also dem Tag des Inkrafttretens des VSBG aufgenommen hat. ${ }^{1}$ Begleitend untersucht das BMJV gem. $\$ 43$ Abs. 2 S. 1 VSBG in einem wissenschaftlichen Forschungsvorhaben die Funktionsweise dieser Allgemeinen Verbraucherschlichtungsstelle. Im Fokus stehen insbesondere Erkenntnisse in Bezug auf die Inanspruchnahme, Fallzahlen, Arbeitsweise, Verfahrensdauer, Erfolgsquoten, Kosten und Entgelte der Stelle.

Das BMJV hat die Autoren, Dr. Naomi Creutzfeldt und Dr. Felix Steffek, mit dem empirisch angelegten Forschungsvorhaben beauftragt. Die Laufzeit des Projekts begann am 1.3.2017 und endet am 31.12.2020. Gemäß $\$ 43$ Abs. 3 Halbs. 2 VSBG war dem Bundestag und dem Bundesrat bis zum 31.12.2018 ein Zwischenbericht vorzulegen. Diesen Zwischenbericht hat die Bundesregierung als BT-Drucks. $19 / 6890^{2}$ dem Bundestag und als Bundesrats-Drucksache $650 / 18^{3}$ dem Bundesrat übermittelt. Der vorliegende Beitrag

1 Siehe www.verbraucher-schlichter.de.

2 Abrufbar unter dipbt.bundestag.de/dip21/btd/19/068/1906890.pdf

3 Abrufbar unter www.bundesrat.de/SharedDocs/beratungsvorgaenge/ 2018/0601-0700/0650-18.html. fasst die zentralen Ergebnisse dieses Zwischenberichts zusammen. Es handelt sich dabei um eine stark erweiterte Fassung des Executive Summary des offiziellen Zwischenberichts. Nach dem Ende des Forschungsvorhabens am 31.12.2020 ist dem Bundestag und dem Bundesrat ein Abschlussbericht gem. $₫ 43$ Abs. 3 Halbs. 1 VSBG zu übermitteln.

Das Forschungsprojekt soll einen Beitrag zur zukünftigen Ausgestaltung der Verbraucherstreitbeilegung in Deutschland leisten. Die politischen Rahmenbedingungen dafür haben sich seit Erlass des VSBG allerdings geändert. Nach der ursprünglichen Konzeption in $\$ 29$ Abs. 2 VSBG können die Länder von der Einrichtung von Universalschlichtungsstellen auf Länderebene absehen, wenn ein ausreichendes Schlichtungsangebot besteht. Ein solches ausreichendes Schlichtungsangebot ist durch die Allgemeine Verbraucherschlichtungsstelle in Kehl zumindest bis zum Auslaufen der Förderung durch das BMJV am 31.12.2019 sichergestellt. Für die Zeit danach begannen die Länder mit der Vorbereitung auf die Einrichtung von Universalschlichtungsstellen gem. $\$ 29$ Abs. 1 VSBG. Im Koalitionsvertrag haben die Regierungsparteien am 12.3.2018 demgegenüber Folgendes beschlossen: ${ }^{4}$ „Die allgemeine Verbraucherschlichtungsstelle wird dauerhaft zentral vom Bund getragen werden. Mit den Ländern sollen Gespräche über eine Beteiligung geführt werden." Vor diesem Hintergrund hat das BMJV am 23.1.2019 den Referentenentwurf eines Gesetzes zur Änderung von Vorschriften über die außergerichtliche Streitbeilegung in Verbrauchersachen veröffentlicht. ${ }^{5}$ Dort ist u.a. die Übertragung der ergänzenden Verbraucherschlichtung auf den Bund vorgesehen.

\section{Forschungsvorhaben und Daten}

Im Zentrum des Forschungsvorhabens steht die Frage danach, wie sich die Verbraucherschlichtung in der Praxis bewährt. Die Studie ist also empirisch ausgerichtet und macht selbst keine Regelungsvorschläge. Gegenstand der Untersuchung sind die Verfahren vor der Allgemeinen Verbrau-

\footnotetext{
4 Koalitionsvertrag zwischen CDU, CSU und SPD vom 12.3.2018, 19. Legislaturperiode, S. 124.

5 Abrufbar unter bmjv.de/SharedDocs/Gesetzgebungsverfahren/DE/
} Streitbeilegung_Verbrauchersachen.html. 
cherschlichtungsstelle am Zentrum für Schlichtung e.V. mit Sitz in Kehl. Die Stelle ist gem. $\$ 4$ Abs. 1 VSBG sachlich für Streitigkeiten aus einem Vertragsverhältnis zwischen einem Verbraucher und einem Unternehmer zuständig. Antragsberechtigt sind nur Verbraucher. ${ }^{6}$ Während der Verbraucher seinen Wohnsitz oder gewöhnlichen Aufenthalt in der Europäischen Union oder dem Europäischen Wirtschaftsraum haben kann, muss der Unternehmer in Deutschland niedergelassen sein. ${ }^{7}$

Das Forschungsvorhaben beruht auf vier Datenquellen: öffentlich zugänglichen Informationen, unmittelbar von der Allgemeinen Verbraucherschlichtungsstelle erhobenen Statistiken, von den Parteien des Schlichtungsverfahrens beantworteten Fragebögen und ergänzend geführten Interviews mit Beteiligten und interessierten Personen. Seit Beginn der Tätigkeit der Allgemeinen Verbraucherschlichtungsstelle am 1.4.2016 bis zum Datenstichtag des Zwischenberichts am 31.7.2018 wurden insgesamt 4.117 Verfahren beantragt. Die dazu erhobenen Grunddaten wurden für den Zeitraum 1.4.2016 bis 31.7.2018 in dem Zwischenbericht ausgewertet. Die geographische Verteilung der antragstellenden Verbraucher folgt in etwa den Bevölkerungszahlen der Bundesländer. Auf Seiten der Unternehmer entspricht die Verteilung über die Bundesländer hingegen nicht immer der Verteilung der Unternehmer in Deutschland.

Vom 1.8.2017 bis zum 31.7.2018 wurden zudem 1.300 Verbraucher und 754 Unternehmer gebeten, einen Fragebogen mit 35 Fragen (Verbraucher) beziehungsweise 32 Fragen (Unternehmer) auszufüllen. Darauf haben 248 Verbraucher und 23 Unternehmer geantwortet. Die Antworten der Verbraucher sind weitgehend repräsentativ in Bezug auf sämtliche von Verbrauchern gestellte Schlichtungsanträge. Eine solche Repräsentativität ist bei Studien dieser Art, zumal im Zeitpunkt eines Zwischenberichts, ungewöhnlich und lässt statistisch gut begründete Aussagen zu. Die Antworten der Unternehmer haben wegen ihrer geringen Zahl hingegen eine noch beschränkte Aussagekraft. Für den Abschlussbericht wird eine Zahl von mindestens 100 Unternehmerantworten angestrebt, auf deren Grundlage dann verlässlichere Aussagen getroffen werden können. Schließlich wurden Interviews sowohl mit Unternehmern als auch Verbrauchern geführt. Zahlenmäßig stehen dabei die Unternehmer im Vordergrund. Durch die Konzentration auf Unternehmer wird insbesondere die geringere Antwortquote der Unternehmer bei den Fragebögen ausgeglichen.

Soweit nicht anders vermerkt beziehen sich die Angaben in dieser Zusammenfassung auf den Jahreszeitraum 1.8.2017 bis 31.7.2018. In diesem Zeitraum stellten 2.217 Verbraucher einen Antrag auf Schlichtung. Die folgende Darstellung folgt den Parteien und der Schlichtungsstelle auf folgenden Etappen durch den Konflikt und das Verfahren: Konfliktsituation vor der Antragstellung (unter III), Verfahrenseinleitung (unter IV), Verfahrensdurchführung (unter V), Verfahrensabschluss und Schritte nach dem Verfah-

$6 \$ 4$ Abs. 1 VSBG; $\$ 1.1$ Verfahrensordnung der Allgemeinen Verbraucherschlichtungsstelle.

$7 \$ 4$ Abs. 4 VSBG; $\$ 1.3$ Verfahrensordnung der Allgemeinen Verbraucherschlichtungsstelle. ren (unter VI) sowie Aufwand (unter VII). Der Beitrag endet mit einem Ausblick (unter VIII).

\section{Konfliktsituation vor der Antragstellung}

Die Verbraucher treten vor allem mit Konflikten aus den Bereichen Waren für Verbraucher, Dienstleistungen im Freizeitbereich, Finanzdienstleistungen, allgemeine Dienstleistungen, Postdienste und elektronische Kommunikation an die Stelle heran. ${ }^{8}$ Typische Beispiele sind Streitigkeiten über Haushaltsgeräte, Waren aus der Informations- und Kommunikationstechnik, Pauschalreisen und Mobilfunktelefondienste. Als wichtigste Ziele nennen die Antragsteller die Lösung des Problems (86\%), eine finanzielle Kompensation $(77 \%)$ und recht zu bekommen ohne dabei Kosten einzugehen (70\%). Als Wert der Streitigkeit geben die Verbraucher in $78 \%$ der Fälle einen Betrag zwischen 0 und $500 €$ an. Einen Konfliktwert zwischen 500 und $1.000 €$ nennen $9 \%$ der Verbraucher. Es geht also um Konflikte mit einem eher geringeren monetären Streitwert.

Von denjenigen Verbrauchern, die einen Antrag bei der Allgemeinen Verbraucherschlichtungsstelle einreichen, geben $69 \%$ an, die Alternativen der Konfliktlösung zu kennen und die Eigenschaften der verschiedenen Verfahren einschätzen zu können. Hier ist zu bedenken, dass die Kenntnisse und Kompetenzen von Verbrauchern, die ihren Weg nicht zu der Stelle finden, davon stark abweichen könnten.

Wenn kein Schlichtungsangebot zur Verfügung stünde, hätten $54 \%$ der Verbraucher den Weg zu den Gerichten gesucht. Bei nahezu der Hälfte der Verbraucher bestünde ohne das Schlichtungsangebot also die Gefahr, dass kein Verfahren der Konfliktbewältigung genutzt worden wäre. Hier wird deutlich, dass die Verbraucherschlichtung den Zugang zur gerechten Konfliktlösung (access to justice) eröffnet.

Zur Allgemeinen Verbraucherschlichtungsstelle finden die Verbraucher im Wesentlichen durch eigene Recherche im Internet (42\%) oder aufgrund von Informationen in den Allgemeinen Geschäftsbedingungen oder auf der Webseite des Unternehmers (21\%). Weitere $15 \%$ der Verbraucher wurden von den Unternehmern auf die Allgemeine Verbraucherschlichtungsstelle hingewiesen. In zusammen- genommen 36 \% der Fälle kam die relevante Information also von Seiten des Unternehmers. Diese Zahl ermöglicht eine erste Evaluierung der neu geschaffenen Informationspflichten der Unternehmer in $\$ \$ 36$ und 37 VSBG.

\section{Verfahrenseinleitung}

Wie bereits dargestellt, gingen seit dem Beginn der Tätigkeit der Allgemeinen Verbraucherschlichtungsstelle am 1.4.2016 bis zum 31.7.2018 4.117 Anträge von Verbrauchern ein. Im Jahreszeitraum 1.8.2017 bis 31.7.2018 wurden 2.217 Anträge gestellt. Von diesen Anträgen wurden 82 Anträge über

8 Produktgruppen in Anlehnung an die COICOP-Klassifikation; dazu näher ec.europa.eu/eurostat/statistics-explained/index.php?title=Glossary:Classification_of_individual_consumption_by_purpose_(COI-

$\mathrm{COP}) /$ de. 
die europäische Online-Streitbeilegungsplattform ${ }^{9}$ eingereicht. Davon betraf die Mehrzahl, nämlich 61 Anträge (rund $74 \%$ ), Konflikte, bei denen sowohl der Verbraucher als auch der Unternehmer in Deutschland ansässig waren. In der Praxis stellen die Verbraucher ihre Anträge in über der Hälfte der Fälle (57 \%) über das Formular auf der Webseite der Stelle. Per Brief leiten $25 \%$ und per E-Mail $17 \%$ der Verbraucher das Verfahren ein. In den Antragstellungen per Brief ist ein Massenantrag enthalten, in dem mehr als 300 gebündelte Schlichtungsanträge im Bereich des grauen Kapitalmarkts eingereicht wurden. Ließe man diese Anträge beiseite, läge der Anteil der elektronisch gestellten Anträge (Webseite, Email) noch deutlich höher.

Im Zeitraum 1.4.2016 bis 31.12.2016 waren $33 \%$ aller Anträge unzulässig ( $11 \%$ aller Anträge wegen Unzuständigkeit der Stelle). Im Zeitraum 1.1.2017 bis 31.12.2017 sank die Zahl unzulässiger Anträge auf $20 \%$ (8 \% aller Anträge wegen Unzuständigkeit). Bemerkenswert ist daran erstens die hohe Quote unzulässiger Anträge und zweitens die Abnahme des Anteils unzulässiger Anträge um 13 Prozentpunkte. Für die Parteien scheint es in einer erheblichen Zahl von Fällen nicht leicht zu sein, die richtige Schlichtungsstelle mit den notwendigen Antragsinhalten anzusprechen. Im weiteren Verlauf des Forschungsprojekts wird zu beobachten sein, ob die zunehmende Vertrautheit der Verbraucher mit der Verbraucherschlichtung zu einem weiteren Absinken der Verweisungs- und Ablehnungsquoten führt.

Die Allgemeine Verbraucherschlichtungsstelle bildet mit anderen Schlichtungsstellen ein informelles Netzwerk, in dem Nutzer auf den richtigen Weg verwiesen werden. Diese Verweisungen erfolgen gegenwärtig jedoch ohne ausdrückliche Regelung im VSBG. Die Verweisungen beschränken sich zudem auf Verweisungen unter den Schlichtungsstellen. Es handelt sich also nicht um ein Konfliktscreening, in dem die Geeignetheit für andere Verfahrenstypen wie die Mediation oder das Gerichtsverfahren geprüft wird.

\section{Verfahrensdurchführung}

Das Schlichtungsverfahren ist für die Parteien nicht zwingend. Sie haben die Gelegenheit zur Stellungnahme und können der Schlichtungsstelle Dokumente zukommen lassen. Die Allgemeine Verbraucherschlichtungsstelle ist neutral. Kern des Verfahrens ist die durch Streitmittler durchgeführte Schlichtung, die auch offen für mediative Elemente ist. Technik kommt nur zur Unterstützung der Tätigkeit der Streitmittler zum Einsatz, wird jedoch nicht zur Lösungsfindung selbst eingesetzt.

Sowohl die Verbraucher (61\%) als auch die Unternehmer (67\%) sind mit dem Verfahren klar überwiegend zufrieden. Etwa ein Viertel beider Gruppen, nämlich $26 \%$ der Verbraucher und $22 \%$ der Unternehmer sind mit der Verfahrensführung nicht zufrieden. Für den Abschlussbericht ist die statistische Auswertung der Frage vorgesehen, ob die Verfahrenszufriedenheit mit dem Verfahrensergebnis zusammenhängt.
Verbraucher und Unternehmer attestieren der Allgemeinen Verbraucherschlichtungsstelle durchweg hohe Kompetenzwerte: eine neutrale Stellung schätzen $91 \%$ der Verbraucher und $78 \%$ der Unternehmer; $90 \%$ der Verbraucher und $78 \%$ der Unternehmer fühlen sich ernstgenommen; ein zutreffendes Problemverständnis erfuhren $85 \%$ der Verbraucher und $67 \%$ der Unternehmer; $85 \%$ der Verbraucher und $67 \%$ der Unternehmer halten die Stelle für kompetent und schließlich vertrauen $85 \%$ der Verbraucher und $78 \%$ der Unternehmer der Allgemeinen Verbraucherschlichtungsstelle in Kehl.

Im Vergleich $\mathrm{zu}$ einem Gerichtsverfahren stimmen beide Gruppen darin überein, dass das Verfahren vor der Allgemeinen Verbraucherschlichtungsstelle einfacher (Verbraucher: $89 \%$, Unternehmer: $89 \%$ ), schneller (Verbraucher: $87 \%$, Unternehmer: $100 \%$ ) und kostengünstiger (Verbraucher: $92 \%$, Unternehmer: $89 \%$ ) ist.

Hinsichtlich der Verfahrensdauer berichten $60 \%$ der Verbraucher und $56 \%$ der Unternehmer eine Dauer von unter einem Monat, $36 \%$ der Verbraucher und $33 \%$ der Unternehmer notieren eine Dauer von einem Monat bis zu drei Monaten und nur $4 \%$ der Verbraucher und $11 \%$ der Unternehmer berichten eine längere Dauer. Mit der Dauer zufrieden sind $92 \%$ der Verbraucher und 78 \% der Unternehmer.

\section{Verfahrensabschluss und Schritte nach dem Verfahren}

Das Schlichtungsverfahren kann auf drei Wegen zu einem materiellen Ergebnis führen. Erstens können sich die Parteien außerhalb des Schlichtungsverfahrens einigen (Einigung ohne Teilnahme an der Schlichtung). Zweitens können sich die Parteien innerhalb des Schlichtungsverfahrens einigen und dabei die Anleitung durch die Schlichtungsstelle nutzen, ohne dass diese einen Schlichtungsvorschlag erteilt (Einigung mit Teilnahme an der Schlichtung ohne Schlichtungsvorschlag). Drittens ergeht ein Schlichtungsvorschlag dann, wenn die Parteien an dem Verfahren vollständig teilnehmen, sich letztlich jedoch nicht einigen können (Teilnahme an der Schlichtung ohne Einigung und mit Schlichtungsvorschlag).

Demgegenüber kann ein materielles Ergebnis schon daran scheitern, dass der Antrag des Verbrauchers abgelehnt wird (etwa wegen Unzulässigkeit) oder die Stelle das Verfahren wegen eines Hindernisses abbricht. Zudem können sowohl der Verbraucher als auch der Unternehmer ihre Beteiligung an dem Verfahren zu jeder Zeit ablehnen.

Im Zeitraum 1.8.2017 bis 31.7.2018 wurden 2.210 Anträge formal abgeschlossen. In 1.929 Fällen (87 \%) kam es weder zu einer vollständigen Verfahrensdurchführung noch zu einer Einigung außerhalb des Verfahrens. Der Begriff vollständige Verfahrensdurchführung ist dabei in dem Sinne zu verstehen, dass wechselseitige Stellungnahmen ausgetauscht wurden und das Verfahren nicht vorzeitig beendet wurde.

9 Siehe ec.europa.eu/consumers/odr/main. 
In den 1.929 Fällen sind also auch solche Fälle enthalten, in denen das Verfahren zwar begonnen und möglicherweise auch teilweise durchgeführt, dann aber nicht vollständig abgeschlossen wurde. Bei strikter Betrachtung kam es nur in 19 Fällen, also rund $1 \%$ aller Anträge, zu einer vollständigen Verfahrensdurchführung. Zu einem Schlichtungsvorschlag samt Einigung kam es nur in 13 Verfahren.

Formal erfolgreich im Sinne einer Einigung innerhalb oder außerhalb des Verfahrens waren im relevanten Zeitraum 281 Fälle (12,7 \%). Lässt man allerdings die 508 Anträge außer Betracht, in denen die Stelle nicht tätig werden konnte (etwa wegen Unzulässigkeit), ergibt sich eine angepasste Ausgangszahl von 1.702 abgeschlossenen und grundsätzlich schlichtungsgeeigneten Fällen. Auf dieser Grundlage steigt die formale Erfolgsquote auf 16,5\%.

Bei der bisherigen Berechnung der formalen Erfolgsquote ist die große Zahl von 1.402 Verfahren, in denen sich der Unternehmer nicht am Verfahren beteiligt, zu Lasten der Quote mitberücksichtigt. Die Unternehmerbeteiligung ist insbesondere deshalb niedrig, weil es sich um ein freiwilliges, aber für Unternehmer kostenpflichtiges Verfahren handelt. Lässt man diese 1.402 Fälle mangelnder Unternehmerbeteiligung zudem außer Betracht, ergibt sich eine qualifiziert angepasste Ausgangszahl von 300 Verfahren. Auf dieser Grundlage ergibt sich bei 281 formal erfolgreichen Verfahren eine Erfolgsquote von 93,7 \%.

Bei der Interpretation dieser Verhältnisse ist auch zu bedenken, dass zahlreiche Konfliktlösungen nur deshalb zustande kommen, weil der Verbraucher sich vor der Verfahrenseinleitung an den Unternehmer wenden muss ${ }^{10}$ und weil die Parteien nach Antragstellung in einen Dialog treten. Konfliktlösungen, die deshalb zustande kommen, weil sich der Verbraucher mit Blick auf ein mögliches Schlichtungsverfahren vor der Antragstellung an den Unternehmer wendet, werden im Rahmen dieser Studie nicht statistisch erfasst. Der Befund erinnert in gewisser Hinsicht an die Situation in der Zivilgerichtsbarkeit, wo das abschließende streitige Urteil in der Gesamtschau nicht der Regelfall ist.

Betrachtet man nicht den formalen, sondern den materiellen Verfahrenserfolg, tritt die Ergebnisakzeptanz in den Fokus. $50 \%$ der Verbraucher sind bereit, das Ergebnis zu akzeptieren, $22 \%$ der Verbraucher sind unentschieden und $28 \%$ der Verbraucher lehnen das Ergebnis ab.

Kommt es nicht zu einer Einigung, will die Mehrzahl der Verbraucher (66\%) und der Unternehmer (56\%) die Beschwerde weiterverfolgen. Anders als die Unternehmer (11\%) ziehen die Verbraucher (39\%) dabei eher den Weg in die weitere außergerichtliche Konfliktlösung vor. Demgegenüber ziehen $45 \%$ der Verbraucher und $33 \%$ der Unternehmer den Gang zu Gericht in Betracht.

$10 \$ 4.2$ Verfahrensordnung der Allgemeinen Verbraucherschlichtungsstelle.

$11 \$ 11$ S. 2 Verfahrensordnung der Allgemeinen Verbraucherschlichtungsstelle; ein Verfahrensentgelt fällt für Verbraucher nur bei missbräuchlicher Antragstellung an.

\section{Aufwand}

Das Verfahren vor der Allgemeinen Verbraucherschlichtungsstelle ist für die Verbraucher grundsätzlich kostenlos. ${ }^{11}$ Im Zeitraum 1.8.2017 bis 31.7.2018 sind 83 \% der Verbraucher vor diesem Hintergrund nur 0 bis $20 €$ an darüberhinausgehenden Kosten pro Verfahren entstanden (z.B. eigene Kosten für Beratung und Kommunikation). Weitere $6 \%$ der Verbraucher berichten von Kosten zwischen 20 und $40 €$. In der Gesamtschau entstehen den Verbrauchern in der großen Mehrzahl also nur geringe Kosten. Dementsprechend empfinden 90 \% der Verbraucher die Kosten des Verfahrens als angemessen.

Die Unternehmer, die sich freiwillig für eine Teilnahme an dem Verfahren entscheiden, haben der Stelle ein Entgelt zu bezahlen. Die Entgelte sind in der Kostenordnung der Stelle geregelt und nach Streitwerten gestaffelt. ${ }^{12}$ In denjenigen Fällen, in denen die Unternehmer sich mit den Verbrauchern nach Antragstellung auf eine Lösung einigen, lehnen die Unternehmer häufig die Teilnahme an dem Verfahren ab. Dies hat den Effekt, dass zwar eine konsensuale Lösung erzielt wird, der Unternehmer mangels Verfahrensteilnahme aber kein Entgelt an die Stelle bezahlen muss. 56 \% der Unternehmer halten die Kosten des Verfahrens für angemessen, während $44 \%$ dem widersprechen. Stellungnahmen der Unternehmer in den Interviews deuten darauf hin, dass die Kosten des Verfahrens vor allem bei geringen Konfliktwerten als unattraktiv empfunden werden.

Den Zeitaufwand gemessen in Arbeitsstunden beziffern $60 \%$ der Verbraucher auf 0 bis 5 Stunden, während die nächsten $20 \%$ einen Aufwand von 5 bis 10 Stunden nennen. Die Unternehmer berichten zu $42 \%$ einen Zeitaufwand von 0 bis 2 Arbeitsstunden und ebenfalls zu $42 \%$ von 2 bis 4 Stunden. Die Allgemeine Verbraucherschlichtungsstelle selbst geht von einem Zeitaufwand von 4 bis 5 Stunden pro Fall aus. Davon entfallen etwa drei Stunden auf Qualitätssicherungsmaßnahmen, die das VSBG vorschreibt. Der Zeitaufwand für ein vollständig durchgeführtes Verfahren liegt nach Schätzung der Stelle zwischen 3,5 und 40 Stunden.

\section{Ausblick}

Die Verbraucherschlichtung erfolgt in Deutschland seit noch nicht einmal zwei Jahren unter den Rahmenbedingungen des VSBG. Der Ende Dezember 2018 vorgelegte Zwischenbericht über das Forschungsvorhaben zur Funktionsweise der Allgemeinen Verbraucherschlichtungsstelle in Kehl gewährt einen ersten empirischen Einblick in die Wirklichkeit der Verbraucherschlichtung. Dabei ist zu bedenken, dass das hier vorgestellte Forschungsvorhaben auf die Allgemeine Verbraucherschlichtungsstelle in Kehl fokussiert ist und die Ergebnisse nicht ohne weiteres auf andere

12 Die Kostenordnung ist abrufbar unter www.verbraucher-schlichter.de/ schlichtungsverfahren/kostenordnung. 
Aspekte und Institutionen der Verbraucherschlichtung übertragen werden können.

Die bisher vorliegenden Daten deuten darauf hin, dass sich die Verbraucherschlichtung in der Praxis bewährt und einen niedrigschwelligen Zugang zur Konfliktlösung ermöglicht. Die geringe Teilnahmebereitschaft auf Seiten der Unternehmer wirft die Frage auf, warum diese oft ein Verfahren ablehnen, dessen Kompetenz und Vertrauenswürdigkeit die klare Unternehmermehrheit schätzt und das im Vergleich zum Gerichtsverfahren als einfacher, schneller und kostengünstiger geschätzt wird.

In die Laufzeit des Forschungsvorhabens fällt nach gegenwärtigem Stand des Referentenentwurfs zur Reform der außergerichtlichen Streitbeilegung in Verbrauchersachen der Übergang der ergänzenden Verbraucherschlichtung auf den Bund und der Beginn der Tätigkeit einer bundesweiten Universalschlichtungsstelle ab 1.1.2020. Darüber hinaus werden die bis zum Abschluss der Forschung zum 31.12.2020 erhobenen weiteren Daten neue Erkenntnisse ermöglichen. Insbesondere ist geplant, mit statistischen Korrelationsverfahren ein besseres Verständnis der Verbraucherschlichtung dadurch zu gewinnen, dass bisher unverbundene Datengruppen miteinander in Beziehung gesetzt werden.

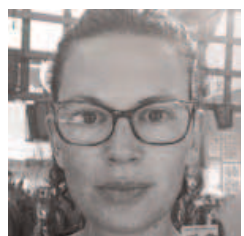

Dr. Naomi Creutzfeldt

Senior Lecturer, Law School, University of Westminster N.Creutzfeldt@westminster.ac.uk

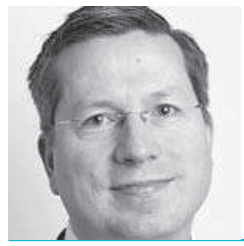

Dr. Felix Steffek LL.M. (Cambridge)

University Lecturer, Faculty of Law, University of Cambridge; Senior Member, Newnham College

fs256@cam.ac.uk

\section{Kirsten Schroeter und Silke Freitag}

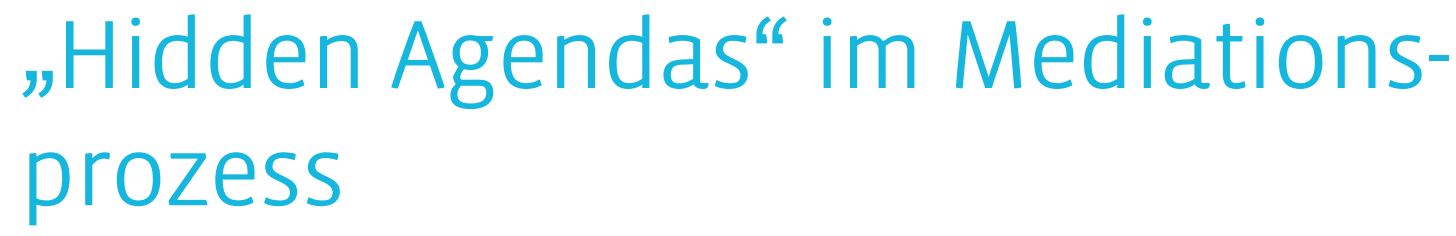

Der Beitrag beschreibt die haltungsbezogenen und methodischen Herausforderungen für Mediatoren im Umgang mit bewusst verdeckten Motiven von Mediationsparteien sowohl in der Auftragsklärung wie im Mediationsverlauf. Die Autorinnen plädieren für eine akzeptierende Haltung gegenüber „hidden agendas“. Sie beschreiben anhand von mehreren Fallbeispielen den methodischen Dreischritt im Umgang mit „hidden agendas“: 1 . Intuitionen über Verborgenes, 2. Spekulationen über Unausgesprochenes, 3. Separate Konfrontation mit der „Wahrheit der Situation".

\section{A. Bedeutung und Herausforderungen}

Wer als Mediatorin sinnvolle Arbeit leisten möchte, muss sicherstellen, dass die grundlegenden Rahmenbedingungen hierfür gegeben sind. Das bedeutet aus unserer Sicht, dass der Fall für Mediation geeignet sein sollte und das Verfahrensdesign fallspezifisch angepasst ist. Zudem sollte das Unterstützen von Klärungen im mediativen Sinne möglich er- scheinen: Ein gemeinsames Gespräch, in dem mindestens Klarheit und eine konsequente Orientierung an den Interessen aller Beteiligten gewährleistet sind. Dies setzt wiederum voraus, dass die Beteiligten ihre Interessen aktiv einbringen.

Wenn Konfliktparteien sich in einer Mediation nun in einem bewussten Willensakt dafür entscheiden, nicht alles offenzulegen, sprechen wir von einer "hidden agenda“. Dass Parteien nicht alles zur Sprache bringen möchten, was ihnen wichtig ist, erachten wir durchaus als Normalfall in Mediationen und insofern nicht zwingend als problematisch. „Hidden agendas“ können jedoch explizite Fallstricke für die Auftragsklärung oder den Fortgang einer Mediation insbesondere beim Aushandeln einer Vereinbarung - darstellen, wenn trotz systematischer Interessenserhellung und (scheinbarer) Verständigung der Parteien kein Konsens gefunden wird und eine unverständliche Blockadesituation entsteht. 